\title{
Mechanical Performance of Montmorillonite Dispersed Jute Reinforced Composite
}

\author{
Muhammad Hasibul Hasan ${ }^{1, a^{*}}$ and Md. Sazib Mollik ${ }^{2}$ \\ ${ }^{1,2}$ Department of Manufacturing \& Materials Engineering, International Islamic University Malaysia, 50728, Kuala Lumpur, Malaysia
}

\begin{abstract}
Surface treated jute accomplished by chemical treatments which enhanced the adhesion between polyester resin and modified surface within the composite. Baking time for 6 hours also reduced the moisture content and diminishes the hydrophilic properties of the corchorus olitorius jute fiber. Montmorillonite shell type nanoclay was dispersed $1 \%, 3 \%$ and $5 \%$ within the jute fiber polyester matrix to enhance the mechanical performance. Effect of temperature and high humidity were evaluated for this nanoclay filled composite through hydrothermal test for 15 days in the environmental chamber. Environmental degradation was not remarkable due to the exposure of the temperature $80^{\circ} \mathrm{C}$ and $95 \% \mathrm{RH}$ for this time period. Ductile properties like yield strength (YS), $\%$ of elongation were calculated for two different stoke rate to understand the strain rate effect. $1 \%$ addition of nanoclay within the composite shows the better performance in terms of yield, flexural and impact strength while $5 \%$ dispersed of nanoclay does not have any beneficial effect within the composite due to the density and non-homogeneous mixture of the clay. Fracture morphology by SEM/ FESEM revealed voids, broken fibers and nano particles within the matrix.
\end{abstract}

\section{Introduction}

Over the past couple of decades, natural reinforced composites have fascinated substantial attention as a potential structural material. Jute is one of the vital agrofibers (lignocellulosic fiber) which draw worldwide consideration as a potential reinforcement of polymer composite because of its natural properties such as low density, high tensile modulus and low elongation at break. A hindrance to natural fibre composites is the reduction of mechanical properties these composites absorb moisture and the water molecules can act as plasticizers by simultaneously influencing the fibres, the matrix and the interface, thus creating regions of poor transfer efficiency $[1,2]$. In this situation, moisture absorption and water absorption can be protected using nano-clay because nano-clay is one of the most affordable materials out of those showing promising results in polymers. Nano-clay improves materials' stiffness, strength, fatigue resistance and thermal stability. The high aspect ratio of nano-clay results in a larger interfacial area which greatly improves its reinforcement properties. Nano-clay enhances the elastic modulus of the hybrid composites [3].

In this study, Bangla tossa special jute and different weight percentages of nano-clay were used. This fiber was chosen because it has some special characteristics due its extra strength, such as its fibre being silkier, stronger and softer than other grades of jute.
To the best of the authors' knowledge, this is the first time Bangla tossa special jute and naturally occurring inorganic nano-clay have been introduced into the composite system for the purpose of producing a material with better mechanical and environmental stability.

\section{Material and methods}

\subsection{Fiber treatment and Composite processing}

Tossa jute fibers (Corchorus olitorius) of Bangla tossa special grade were obtained from Bangladesh Jute Research Institute (BJRI), Bangladesh. There were another three matrix materials with reinforced material that were used such as polyester resin, hardener (methyl ethyl ketone peroxide) and nano-clay (montmorillonites). It is principally suitable for hand lay-up due to its low viscosity and non-thixotropic nature. Jute fibres were cut to $30 \mathrm{~cm}$ in length and were soaked in a $5 \% \mathrm{NaOH}$ solution at $30^{\circ} \mathrm{C}$. The fibres were kept immersed in the $\mathrm{NaOH}$ solution for 2, 4, 6 and $8 \mathrm{~h}$. The fibres were then washed several times with fresh water to remove any $\mathrm{NaOH}$ on the fibre surface and finally washed again with distilled water. The fibres were then dried at room temperature for $48 \mathrm{~h}$ and subsequently dried using ovendrying at $70^{\circ} \mathrm{C}$ for 6 hours. Jute polyester composites containing raw jute with nano-clay and jute fibres were fabricated by hand lay-up process in the form of a square

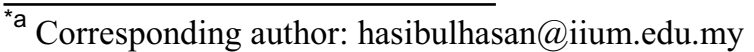


shape of size $32 \mathrm{~mm}$ as their length, $32 \mathrm{~mm}$ as their width and $3 \mathrm{~mm}$ as their thickness. The polyester resin to hardener mixing ratio was $25 \%$ wt to $5 \%$ wt. There were several nano-clay ratios ued, i.e. $1 \% \mathrm{wt}, 3 \% \mathrm{wt}$ and $5 \% \mathrm{wt}$. The jute fibers ratio was $70 \%$ for every specimen. All specimens are fabricated individually to avoid voids and cutting effects during machining.

\subsection{Tensile tests and simulation}

Tensile tests were performed according to ASTM 412 type 4 by using a Lloyd 10KN Universal Testing Machine. The shape and dimensions of the specimens are $115 \mathrm{~mm} \times 19.42 \mathrm{~mm} \times 3 \mathrm{~mm}$. The ductile properties, namely tensile strength, Young's modulus, and strain at break values, were recorded at a stroke rate of $0.8 \mathrm{~mm} / \mathrm{min}$ and $0.5 \mathrm{~mm} / \mathrm{min}$. The Finite Element Analysis (FEA) of jute composite is analyzed in this study. This analysis is actually for achieving the optimum model design. The dog bone sample is analyzed as a 2-D axisymmetric model.

\subsection{Flexural and Impact test}

Samples were tested according to the Test Method-I Procedure A in the standard ASTM D790. Dimensions were $125 \times 12.7 \times 3 \mathrm{~mm}$. The support span and the rate of crosshead motion were $48 \mathrm{~mm}$ and $2 \mathrm{~mm} / \mathrm{min}$, respectively. In this research charpy impact test was carried out according to ASTM D256 by using Dynisco Polymer Test machine. V-notched samples had dimensions of $55 \times 10 \times 10 \mathrm{~mm}$, respectively.

\subsection{Scanning electron microscopy and FESEM}

Scanning electron microscopy was performed on small pieces taken from the tested samples using a low voltage scanning electron microscope. The fracture surfaces of impact samples were coated with a thin layer of gold before because to avoid electrostatic charging and poor image resolution. Fracture surface after tensile testing were experimental using a field emission SEM under a voltage of $10 \mathrm{KV}$.

\section{Result and discussions:}

\subsection{Tensile Properties:}

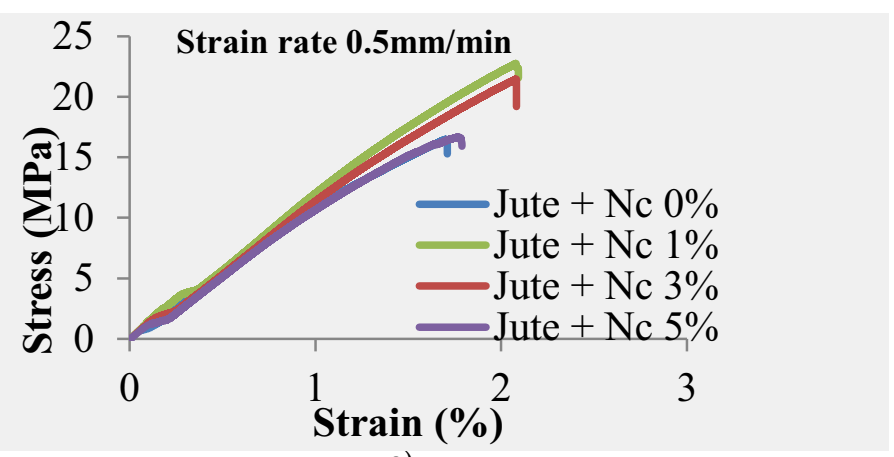

a)

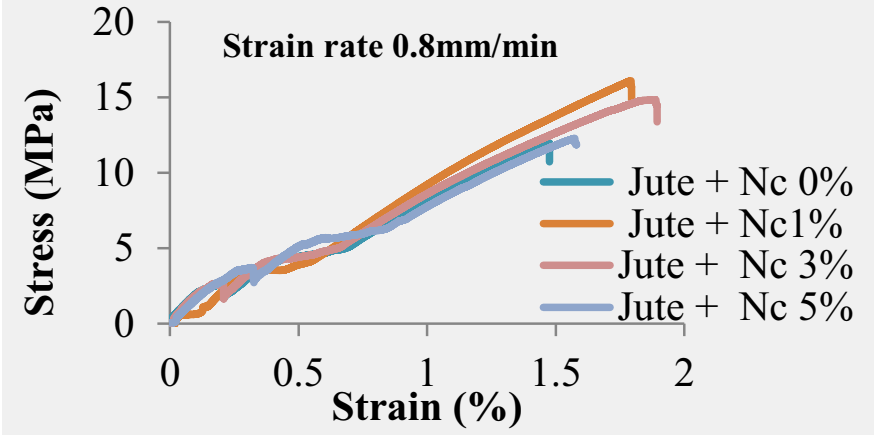

b)

Fig. 1: (a, b) Tensile stress of jute composite

Ductile parameters under different strain rate were calculated via tensile test. From tensile test Figure 1(a) it was observed that the tensile test was carried out $0.5 \mathrm{~mm} / \mathrm{min}$ stroke rate considered as slow stroke rate. Tensile stress was around $22.4 \mathrm{MPa}$ for $1 \%$ additional nanoclay in the tossa jute composite and stress was linearly increased from $4.37 \mathrm{MPa}$ to $22.4 \mathrm{MPa}$. Tossa jute samples $5 \%, 3 \%$ and $0 \%$ nanoclay showed maximum tensile stress nearly $16.6 \mathrm{MPa}, 21.5 \mathrm{MPa}$ and $16.1 \mathrm{MPa}$ respectively. $5 \%$ additional nanoclay clearly showed no benefit to the tensile stress for jute composite may be due to mechanical properties and adding more nanoclay. Fu and Naguib (2009) had studied that, the mechanical properties of tensile strength decreased due to additional of excessive nanoclay. They also observed that, excessive nanoclay was not well scatter in the polymer composite [4]. It was observed form Figure 1(b) that, the tensile test was carried out $0.8 \mathrm{~mm} / \mathrm{min}$ stroke rate considered as medium stroke rate. The tensile stress is highest at room temperature $(15.9 \mathrm{MPa})$ for $1 \%$ addition of nano-clay in the tossa jute composite and linearly increases from $4.4 \mathrm{MPa}$ to $15.9 \mathrm{MPa}$. Moreover, tensile stress of nanclay $0 \%$, nanoclay $3 \%$ and nanoclay $5 \%$ filled composite was around $11.9 \mathrm{MPa}, 12.5 \mathrm{MPa}$ and $14.8 \mathrm{MPa}$ respectively. The range of the tensile strength obtained in the current work is $15.9 \mathrm{MPa}$, which is lower than the range obtained in previous result $(22.4 \mathrm{MPa})$ using the same matrix and fiber material for $1 \%$ additional of nanoclay due to increase of stroke rate as oppose to the $0 \%, 3 \%$ and $5 \%(11.9 \mathrm{MPa}, 12.5 \mathrm{MPa}$ and $14.8 \mathrm{MPa})$ nano-clay is also lower than the range obtain in previous results (16.1MPa, $21.5 \mathrm{MPa}$ and $16.6 \mathrm{MPa}$ ). $5 \%$ addition of nanoclay clearly showed no benefit to the tensile stress for jute composite.

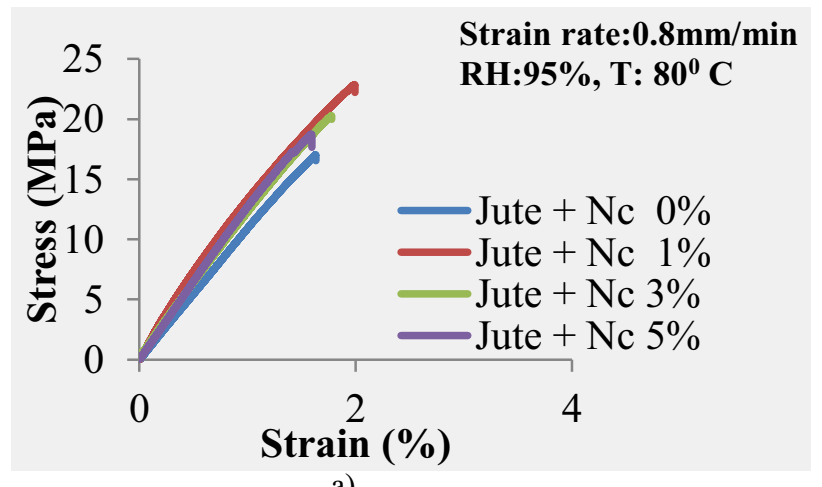

a) 


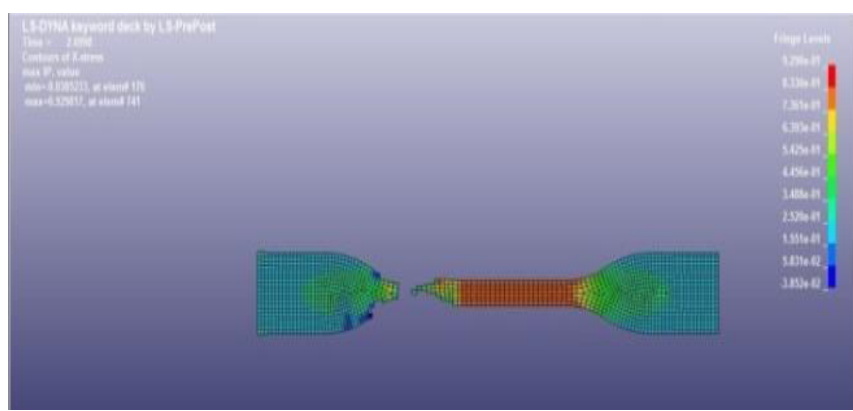

a)

Fig. 2: (a, b) Tensile stress of jute composite after hygrothermal and $\mathrm{X}$-Y plane effect

Environmental degradation due to temperature and humidity was studied through hygrothermal test for 15 days. After the exposure of $800 \mathrm{C}$ temperature and $95 \%$ $\mathrm{RH}$, same samples were carried out tensile test following $0.8 \mathrm{~mm} / \mathrm{min}$ stroke rate. From Figure 2(a) tensile strength increased by $12.7 \%, 20.7 \%$, and $34.4 \%$, for $5 \%, 3 \%$ and $1 \%$ additional of nano-clay respectively. Since fibers were baked at $600 \mathrm{C}$ for 6 hours, which substantially reduced the moisture content with in the fiber. As discussed earlier $8 \sim 12 \%$ moisture content in the fiber gives best performance for the tensile strength. Initial baking may reduce the moisture content of samples around 95\% humidity may increase the humidity to the optimum level which may be the reason of higher tensile strength. Minimum developed stress was around 1.161e-02 $01 \mathrm{~N} / \mathrm{mm} 2$ and maximum stress was found $1.144 \mathrm{e}-$ $01 \mathrm{~N} / \mathrm{mm} 2$ as shown in the Figure 2(b). Stress distribution on $\mathrm{X}-\mathrm{Y}$ plane from minimum to maximum value is identified from fringe level provided in the right corner of the Figure 2(b). Figure b shows that, maximum stress is developed at the middle of the elements as cross section area is small in that location. During $\mathrm{X}-\mathrm{Y}$ plane maximum stress is representing at gage length section.

\subsection{Flexural and Impact test}

From Figure 3(a) shows that, the flexural strength of nano-clay $1 \%$ filled composite was increased $37.29 \%$ which was higher than nano-clay $0 \%$. The flexural strength of nanoclay $3 \%$ filled composite decreased $9.5 \%$ from nanoclay $1 \%$ filled composite according to the Figure 3(a). The same issues and reasons that are specified above are also accountable for nanoclay5\% filled composite. Additional 5\% nano-clay filled composite's flexural strength decreased from 3\% filled nano-clay nearly $27.9 \%$. The flexural strength of nanoclay $1 \%$ filled composite was around $136 \mathrm{MPA}$ as oppose to the flexural strength of nano-clay $0 \%, 3 \%$ and $5 \%$ was 85.28MPa, $124.22 \mathrm{MPa}$ and $89.55 \mathrm{MPa}$ respectively. The range of the flexural strength obtained in the current work is $85.28-136 \mathrm{MPa}$. On the other hand, the flexural modulus of jute composite system and it is observed that, the flexural moduli for all the jute composites are increased. $1 \%$ nano-cli "ed composite shows the maximum value $0.051 \mathrm{G}$ a) lowed by $0 \%, 3 \%$ and $5 \%$ nano-clay filled composite which are $0.04 \mathrm{GPa}$, $0.0455 \mathrm{GPa}$ and $0.048 \mathrm{GPa}$ respectively. Figure $3(\mathrm{~b})$ shows impact strength of jute composites where nano-clay 5\% shows $9.296 \mathrm{~kJ} / \mathrm{m} 2$ which is the minimum value among nano-clay $1 \%$ and nano-clay $3 \%$.

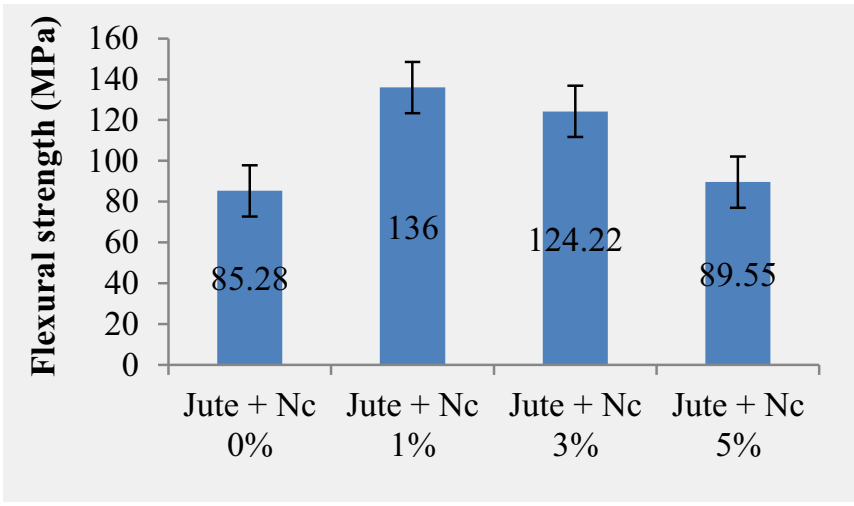

a)

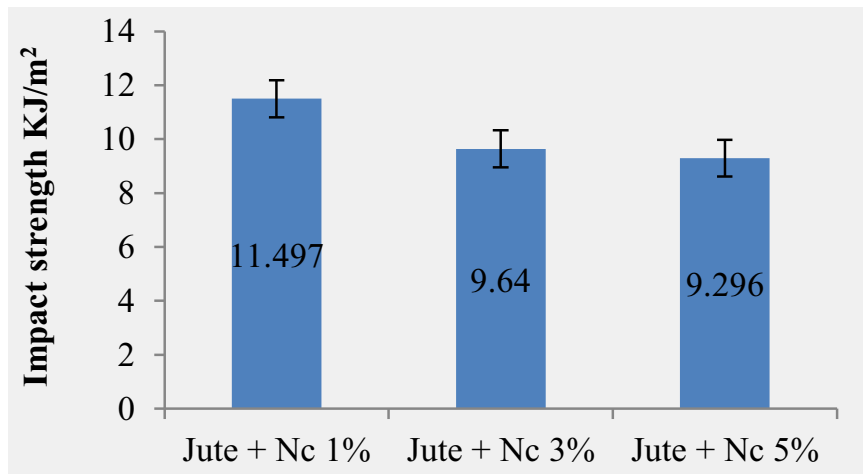

b)

Fig. 3 :( a, b) Flexural and Impact strength of jute composite

\subsection{SEM/FESEM}

Efforts were made of characterize the morphology of the broken surface of jute composite in molecular level.

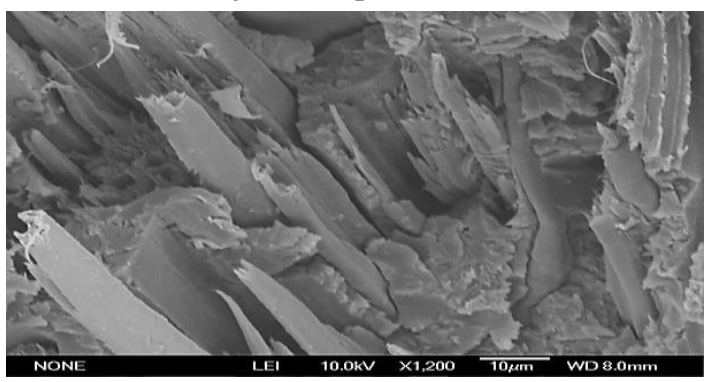

a)

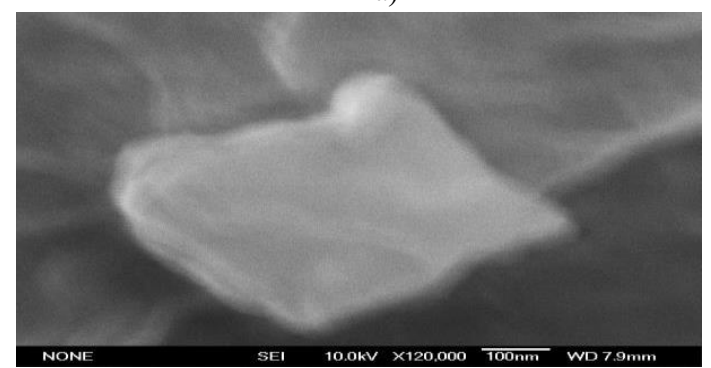

b)

Fig.4: FESEM of nano-clay filled tested samples $a$ and $b$ is the nano particle in the matrix system 
Figure 4 shows the FESEM of broken samples before undergoing thermal analysis. Fractured jute fibers are clearly visible at $1 \%$ nano-clay filled samples and nanoparticle is identified within the matrix.

\section{Acknowledgement}

The authors are gratefully to International Islamic University Malaysia (IIUM) for supporting through grant number FRGS-141310732.

\section{References}

1. Ray, D., et al. "The mechanical properties of vinylester resin matrix composites reinforced with alkali-treated jute fibres." Composites Part A: applied science and manufacturing 32.1 (2001): 119 127.

2. Yang HS, Kim HJ, Park HJ, Lee BJ, Hwang TS. Water absorption behavior and mechanical properties of lignocellulosic filler-polyolefin bio-composites. Comp Struct 2006;72:429-37.

3. Ray, D., et al. "The mechanical properties of vinylester resin matrix composites reinforced with alkali-treated jute fibres." Composites Part A: applied science and manufacturing 32.1 (2001): 119127.

4. Fu, Jin, and Hani E. Naguib. "Effect of nanoclay on the mechanical properties of PMMA/clay nanocomposite foams." Journal of cellular plastics 42.4 (2006): 325-342. 